\title{
Mit Innovationskraft aus der Krise
}

Die 2020er-Jahre begannen nicht so, wie wir es uns alle erhofft haben. Anstatt weiter Wirtschaftswachstum und Wohlstand zu generieren, befinden sich viele Industrieunternehmen im Krisenmodus. Die Aus- und Nachwirkungen von COVID-19 - etwa Angebots- und Nachfrageschwankungen, instabile Lieferketten und Arbeitskräftemangel - lassen sich auf vielen Produktmärkten beobachten. Hinzu kommen neuerdings volatile Finanzmärkte, die zunehmend von Inflationsängsten und politischen Risiken geprägt sind.

Die komplexe Situation ist eine der größten betrieblichen Herausforderungen der letzten Jahre. Viele Unternehmen hat das dazu bewegt, sich technologisch wie organisatorisch neu zu orientieren und Innovationen voranzutreiben. Initiativen zur digitalen Transformation in allen unternehmerischen Bereichen sowie Investitionen in Technologien wie Cloud Computing, Datenanalyse, Internet der Dinge (IoT), Künstliche Intelligenz (KI), robotergesteuerte Prozessautomatisierung, Low-CodeEntwicklung und andere stehen im Mittelpunkt dieser digitalen Evolution. Diese bieten Herstellern fast endlose Möglichkeiten, neue Lösungen zu erkunden, um die betriebliche Effizienz zu steigern und innovative Produkte schneller zu den Kunden zu bringen.

Das ist insofern notwendig, da sich auch die Anforderungen der Kunden schnell weiterentwickeln. Zu deren Augenmerk auf personalisierte Produkte und Services sowie transparente Lieferprozesse kommen zunehmend Nachhaltigkeitsforderungen. Um hierbei das eigene Unternehmen von der Konkurrenz abzuheben und dabei auch global wettbewerbsfähig zu bleiben, ist ein hohes Maß an Agilität und Innovationkraft erforderlich.

Ihre ZWF-Herausgeber und-Redaktion 